\title{
Exploring the Ubiquitin-Proteasome System (UPS) through PROTAC Technology
}

\author{
Carlotta Cecchini ${ }^{\star a}$, Sébastien Tardya ${ }^{a}$ Valentina Ceserania, Jean-Philippe Theurillat ${ }^{\star b}$, \\ and Leonardo Scapozza*a
}

§SCS-DSM Award for best poster presentation in Chemical Biology

\begin{abstract}
In the context of dysregulated ubiquitylation, the accumulation of oncogenic substrates can lead to tumorigenesis. In particular, mutations in Von Hippel-Lindau (VHL) E3 ubiquitin ligase are related to overexpression of hypoxia-inducible factors (HIF-1 $\alpha$ and HIF-2 $\alpha$ ) which is evolving into renal cell carcinoma (RCC). The classical approach of drug discovery focuses on the development of highly selective small molecules able to bind and to inhibit enzymatic active sites. This strategy faces limitations in the context of 'undruggable' proteins, which are challenging to target. The discovery of Proteolysis Targeting Chimeras (PROTACs) as an alternative strategy to induce selective protein degradation is presented as a working hypothesis to understand further the UbiquitinProteasome System (UPS) and eventually counteract RCC cancer lacking VHL ubiquitin ligase.
\end{abstract}

Keywords: Protein degradation · Proteolysis Targeting Chimeras · Renal cell carcinoma - Ubiquitylation

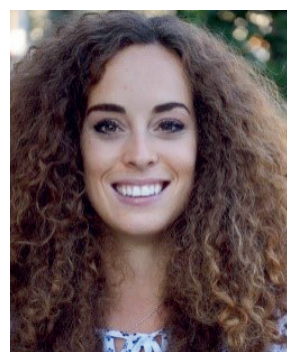

Carlotta Cecchini obtained her Master's degree in Pharmaceutical Sciences in Bologna (Italy), where she was awarded consecutive scholarships for 2015 and 2016 for her excellent results. She performed her Master's research project in Prof. Leonardo Scapozza's group in the School of Pharmaceutical Sciences at the University of Geneva and after receiving her Master's degree in 2017, she began a $\mathrm{PhD}$ in Prof. Leonardo Scapozza's group to continue her research work at the University of Geneva. Carlotta's research focuses on designing and synthesizing heterobifunctional molecules called 'Proteolysis Targeting Chimeras' (PROTACs), which act as targeted degraders to investigate the Ubiquitin-Proteasome System (UPS) in cancers with dysregulated ubiquitylation.

\section{Protein Ubiquitylation and Degradation}

Posttranslational modifications play a fundamental role in protein functions, such as regulation of the folding, interaction with ligands or other proteins and localization to specific subcellular compartments. Moreover, these chemical changes regulate protein functional states, such as signal transduction pathways in the case of signaling proteins or the catalytic activity in the case of enzymes. Among the different posttraslational modifications, ubiquitylation has emerged as an essential player in determining cell function and control, as it can affect the stability, interactions, localization or activity of several proteins. ${ }^{[1]}$ Indeed, proteins that could be harmful to the cell, such as damaged, misfolded or misassembled proteins are finally degraded. [2] Protein degradation is regulated by the Ubiquitin-Proteasome System (UPS) which involves different steps that depend on ubiquitin, a 76 amino acid protein that is highly conserved among higher eukaryotes. The addition of polyubiquitin chains to a target protein serves as a recognition marker and leads to proteolytic degradation through the proteasome. This multiple enzymatic step process consists of a sequential action of ubiquitin-activating enzymes (E1), ubiquitin-conjugating enzymes (E2), and ubiquitin ligases (E3). In particular, E3 ubiquitin ligase recruits an E2 ubiquitin-conjugating enzyme that has been attached to ubiquitin, recognizes a protein substrate, and catalyzes the relocation of ubiquitin from the E2 to the protein substrate, which is finally degraded through the proteasome (Fig. 1). ${ }^{[3]}$

\section{Dysregulations in UPS}

Genetic defects in the ubiquitin system are at the root of many acquired and hereditary diseases, including neurodegen-

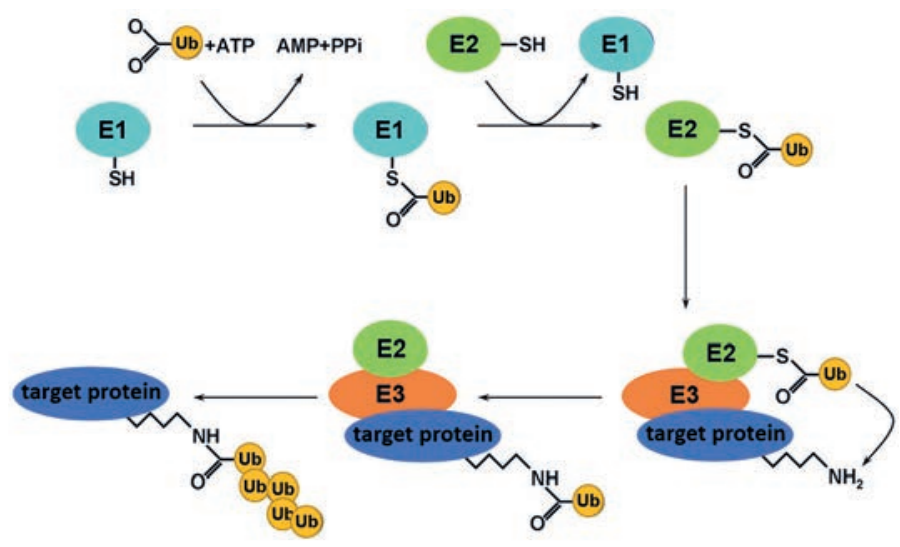

Fig. 1. General scheme representing the ubiquitylation process, adapted from ref. [3]. 
erative, immune, and neoplastic disorders. ${ }^{[4]}$ In cancer, both tumor-suppressing and tumor-promoting pathways are regulated by ubiquitylation. In particular, abnormal expression of E3 ligases is directly associated with altered expression of oncogenic substrates. Depending on the type of ubiquitin ligase, mutations or alterations in their expression can be found in a wide variety of cancers, or less frequently, restricted to a particular type of tumor, thus representing an attractive drug target in cancer. An example is the von Hippel-Lindau tumor suppressor (VHL), a recurrently mutated gene in clear cell renal cell cancer (RCC). [5] VHL encodes for a ubiquitin ligase adaptor protein that forms a Cullin-RING E3 ligase complex with Elongin B \& C, CUL2 and $\mathrm{RBX} 1 .{ }^{[5,6]}$ It is implicated in the recognition and degradation of hydroxyproline modified hypoxia-inducible factors HIF- $1 \alpha$ and $-2 \alpha$ under normoxic conditions (Fig. 2). ${ }^{[7]}$ The loss of VHL function has been shown to promote tumorigenesis by leading to an increased HIF- $1 \alpha$ and $-2 \alpha$ transcriptional activity with consequent upregulation of vascular endothelial growth factor (VEGF) and other factors that are thought to promote survival.[8]

\section{Strategies to Treat RCC Cancer}

Different strategies have been developed to treat RCC tumors, which are often characterized by abnormal vascularization. Small molecule tyrosine kinase inhibitors block receptor signaling by primarily targeting VEGF and platelet-derived growth factor (PDGF), ${ }^{[10]}$ but these agents suffer from low specificity for the targeted kinases. The discovery that the primary genetic event in clear cell RCC (loss of VHL) results in a defect in the UPS suggested that novel therapies targeting this pathway could be employed to induce apoptosis in cancer cells. For this reason, 26S proteasome inhibitors, such as Bortezomib, which stabilize proteins that normally contribute to apoptosis, have been used to treat RCC cancer. ${ }^{[11]}$ Bortezomib causes apoptosis of kidney cancer cell lines in vitro, but Phase II clinical trials results were less promising. ${ }^{[12]}$ Its toxicity probably reflects the consequences of nonspecific inhibition of the UPS. ${ }^{[13]}$ Among other treatments, small molecule inhibitors of Hsp90 have been shown to promote the degradation of HIF $\alpha$ proteins in a VHL-independent manner. ${ }^{[14]}$ Moreover, inhibitors of the mTOR pathway, such as Rapamycin, have been shown to reduce HIF- $1 \alpha$ levels and HIF- $1 \alpha$ transcriptional activity. ${ }^{[15]}$ However, all these treatments lack specificity and therefore have non-negligible side effects. In general, ubiquitin E3 ligases are very specific in their interaction with protein substrates, determining the selectivity of the ubiquitination process. ${ }^{[16]}$ Thus, they are attractive targets for drug discovery since their inhibition would be expected to have fewer 'off-target' effects and less toxicity than other inhibitors. ${ }^{[17]}$ One example is represented by the inhibition of the interaction between the p53 tumor suppressor gene and its E3 ubiquitin ligase, MDM2. The p53 gene is often mutated in cancer, and moreover, functional inactivation of p53 can also occur through its increased proteasomal degradation by MDM2, which is overexpressed in sev-

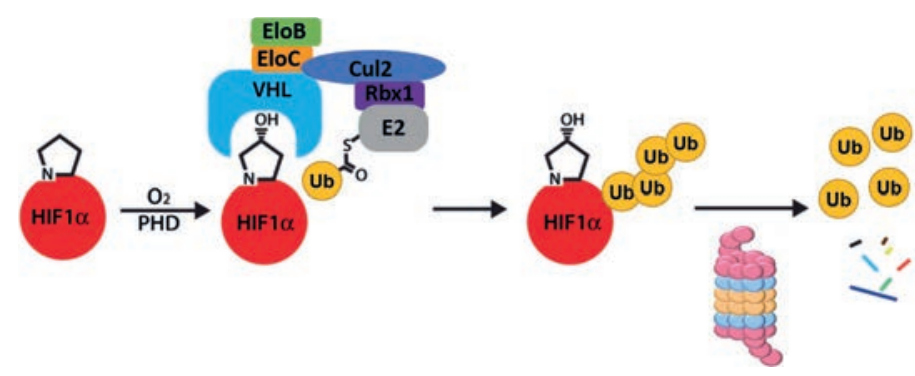

Fig. 2. HIF-1 $\alpha$ ubiquitylation pathway adapted from ref. [9]. Under normoxic conditions, HIF- $1 \alpha$ is constitutively expressed and is recognized by VHL ligase upon hydroxylation by prolyl hydroxylases domain (PHD) enzymes at a specific proline residue. This leads to its subsequent VHLmediated polyubiquination and proteasomal degradation. ${ }^{[9]}$ eral types of tumors. ${ }^{[18]}$ Small-molecule inhibitors called Nutlins occupy the p53 binding site of MDM2, thereby displacing p53 and preventing its degradation. ${ }^{[19]}$ In this way, the p53 tumor suppressor function can be re-established. Unfortunately, translating this strategy to RCC cancer treatment would most likely be ineffective since UPS defects due to VHL mutations lead to a decreased degradation of HIF- $\alpha$ proteins. Therefore, promoting the degradation of HIF- $\alpha$ proteins in RCC cancer needs to be further studied. In this context, the PROTAC approach might represent an attractive strategy to activate the proteasome pathway in a specific manner.

\section{The PROTAC Approach to Explore the UPS in the Context of RCC Cancer}

Among the novel pharmacological strategies for cancer therapy, such as small-molecule inhibitors, monoclonal antibodies and oligonucleotide agents, PROTACs (Proteolysis Targeting Chimeras) have emerged as a potent tool to rapidly and reversibly deplete endogenous proteins. ${ }^{[20]}$

PROTACs are heterobifunctional molecules, which can bind specifically a target protein and induce its degradation through a given E3 ubiquitin ligase acting in a catalytic way. ${ }^{[21]}$ Since this technology benefits from the cell's own protein degradation pathway to specifically remove targeted proteins, it represents an orthogonal approach compared to small-molecule inhibitors, which act through an occupancy-driven pharmacology. ${ }^{[22]}$ Thus, in contrast to small-molecule inhibitors that need a specific cavity or binding pocket which is 'druggable' to lead to therapeutic benefit, PROTACs have been shown to be suitable for targeting substrates that lack an active binding site.[23]

In the context of RCC cancer, the PROTAC approach might be useful to counteract the accumulation of HIF- $1 \alpha$ and $-2 \alpha$, due to the absence of VHL activity, through the recruitment of a different E3 ligase able to degrade them (Fig. 3).

\section{Challenges in PROTAC Design}

PROTAC design relies on the combination of three different chemical moieties: an E3 ligase binder, a target of interest (TOI) ligand and a linker, which connects the two parts (Fig. 4). One of the most common strategies to combine these parts is click chemistry, ${ }^{[25]}$ a reaction under mild conditions that consists of a 1,3-dipolar cycloaddition starting from an azide and alkyne moiety (Fig. 4 and Fig. 5). In addition, the presence of the triazole moiety may represent a metabolic advantage when linked to linear linkers, such as polyethylene glycol (PEG) or alkyl linkers, which are more easily exposed to oxidative metabolism in vivo. [26]

Finding the best combination of the three moieties represents a big challenge in PROTAC development. Firstly, to design and synthesize any new PROTAC, a pharmacologically/biophysically well-characterized ligand, often an inhibitor, is needed as the TOI recruiting moiety. Simultaneously, a known ligand to recruit the E3 ubiquitin ligase is also required. Once the inhibitor for the target protein and a ligand for the E3 ligase have been identified, their functionalization to generate an effective TOI ligand and, respectively, a ligase binder needs careful study of the SAR guided by molecular modeling.[29] This can be explained by the fact that modifications of the ligands might affect the binding affinity for their target. It is also true that a reduced binding affinity does not produce necessarily inactive PROTACs. Indeed, the mechanism of PROTAC-induced degradation is dependent on the formation of a ternary complex and this might be more important in determining a productive PROTAC than the binary affinity of the TOI ligand or the ligase binder, as shown by Crews laboratory in a recent study. ${ }^{[30]}$ Furthermore, different degradation profiles can be observed depending on which E3 ligase is recruited. There are more than 600 E3 ligases predicted in the human genome but so far, only a few have been validated for PROTAC development:[31] in particular VHL, CRBN, MDM2, and cIAP1. In this context, the choice of the E3 ubiquitin ligase is cru- 
Fig. 3. PROTACs can restore the normal ubiquitylation through a catalytic mechanism, adapted from ref. [24].

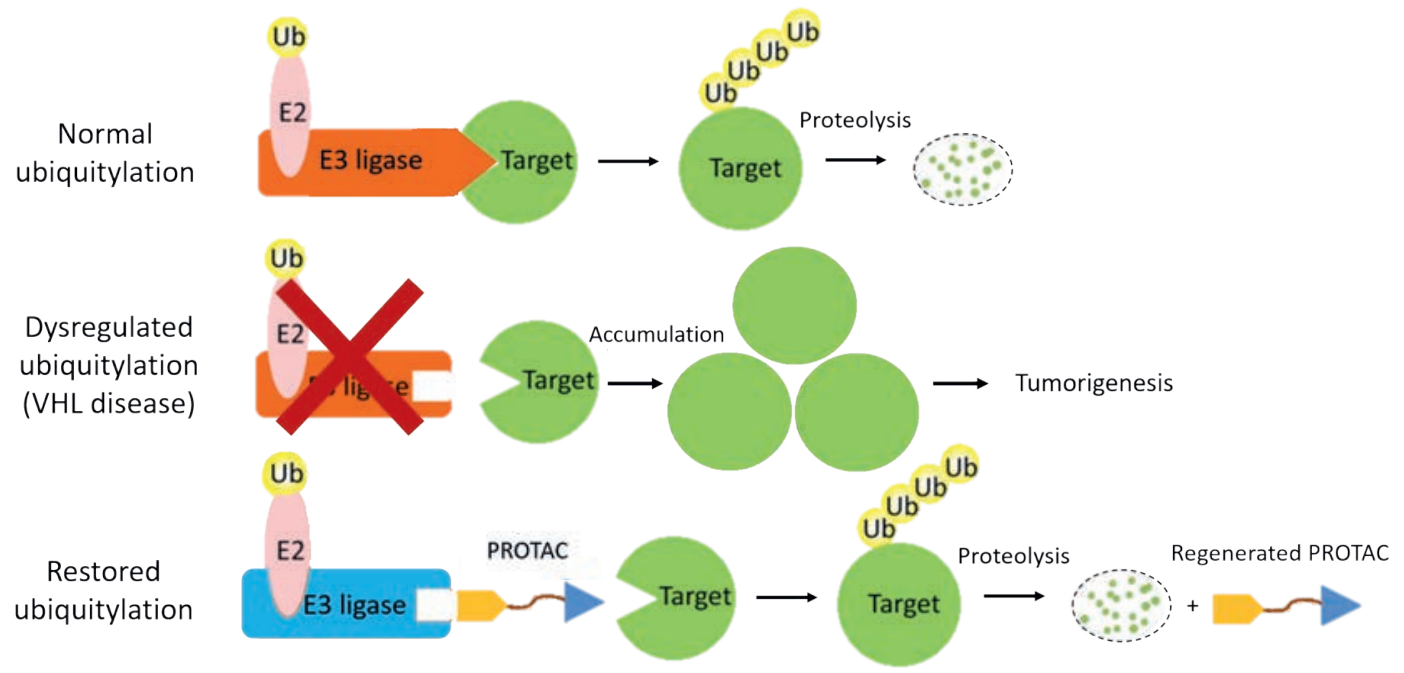

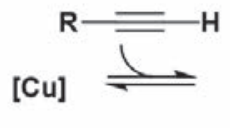

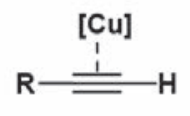<smiles>CC(C)C(C)(C)C</smiles><smiles>[R]C#CC(Cl)(Cl)Cl</smiles><smiles>[R]c1nnn([R])c1[GeH3]</smiles>
[Cu]

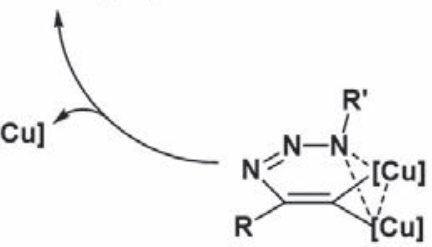

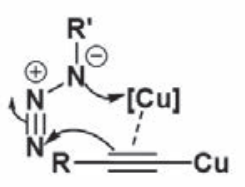

Fig. 5. The copper-catalyzed reaction of an azide with an alkyne forms a 5-membered heteroatom ring: a Cu(I)-catalyzed azide-alkyne cycloaddition (CuAAC). ${ }^{[27]}$. Adapted from ref. [28].

cial. Equally, the species and length of the linkers have a dramatic impact on the activity of the PROTAC and the stability of the ternary complex. In a recent study, Gray and co-workers found that different PROTAC linker lengths favored different ternary complex conformations in which the target protein BRD4 interacts with either the C-terminal or the N-terminal domain of CRBN.[32] This suggests that PROTACs with the same E3 recruiting ligand and TOI ligand can provide different selectivity profiles depending on differences in linker attachment points and linker chemical composition. ${ }^{[32]}$

\section{Hook Effect in PROTACs}

A recurrent issue that can be observed in PROTAC technology is the hook effect, which promotes the formation of ineffective binary complexes (PROTAC-TOI or PROTAC-E3 ligase) at high PROTAC concentrations instead of ternary complexes (TOI-PROTAC-E3 ligase).[33] This phenomenon affects the
PROTAC's potency depending on its concentration and thus it may decrease its activity. Moreover, favorable or repulsive interactions between the TOI and E3 ligase in ternary complex formation might also influence this effect, resulting in a more or less stable ternary complex. ${ }^{[34]}$ The term cooperativity $(\alpha)$ is used to describe these interactions. Positive cooperativity $(\alpha>1)$ takes place when favorable interactions between the TOI and E3 ligase stabilize the ternary complex. In contrast, negative cooperativity $(\alpha<1)$ occurs when repulsive interactions inhibit ternary complex formation. To minimize the extent of the hook effect, positive cooperativity is required, resulting in enhanced productive ternary complex formation. ${ }^{[32]}$ Since these interactions are difficult to predict in advance, minimizing the hook effect is a major challenge. A strategy to reduce this effect might be the progressive release of the two end-moieties of the PROTAC (E3 ligase and TOI binders).

\section{Conclusions}

The recruitment of different E3 ligases through PROTACs might counteract HIF- $1 \alpha$ and $-2 \alpha$ accumulation in RCC tumors. Since certain targets are more responsive to the proteasomal degradation than others, the choice of E3 ubiquitin ligase pairing appears crucial. Additionally, the expression profile of the chosen E3 ligase has an important influence on the degradation of a target in specific tissues or cellular compartments. ${ }^{[35]}$

As mentioned above, there are more than 600 E3 ligases predicted in the human genome but so far, only a few have been explored in targeted protein degradation (CRBN, VHL, IAPs, MDM2, DCAF15, DCAF16, RNF114). [31] Some of them are expressed ubiquitously in different tissues, which may translate to undesired effects of PROTACs. Thus, PROTACs that recruit E3 ligases with a tissue-selective expression profile are expected to present unique opportunities for therapeutic applications, as they should not degrade the targeted protein in tissues where the E3 ligase is not expressed. ${ }^{[36]}$ To assess the tissue-selective expression profile we need to better understand the UPS system using clinical relevant samples and both genetic and chemical perturbation tools such as PROTACs. 
Overall, this technology represents a promising strategy for inducing selective protein degradation in cancer. Some of the challenges in PROTAC design and synthesis were discussed, such as the combination of three different chemical moieties (TOI ligand, linker, ligase binder), the formation of a stable ternary complex, the hook effect and the choice of the recruited E3 ubiquitin ligase. All these aspects are crucial to obtain effective PROTACs, to understand further the Ubiquitin-Proteasome System (UPS) and eventually counteract RCC cancer lacking VHL ubiquitin ligase.

\section{Acknowledgements}

C.C. is grateful to the Swiss Chemical Society and DMS for the best poster award in Chemical Biology. This project received funding from the Swiss National Science Foundation (SNSF, CRSII5_186405/1). We thank D. De Brito Salgado, S. Pannilunghi and C. Terenzi for their help to this research.

Received: February 13, 2020

[1] M. Rape, Nat. Rev. Mo. Cell Biol. 2018, 19, 59, DOI: 10.1038/nrm.2017.83.

[2] H. McDonough, C. Patterson, Cell Stress Chaperones 2003, 8, 303, DOI 10.1379/1466-1268(2003)008<0303:calbtc $>2.0 . c 0 ; 2$.

[3] H. C. Ardley, P. A. Robinson, Essays in Biochem. 2005, 41, 15, DOI: 10.1042/bse0410015.

[4] D. Popovic, D. Vucic, I. Dikic, Nat. Med. 2014, 20, 1242, DOI: 10.1038/ nm.3739.

[5] J. William G. Kaelin, Ann. Rev. Pathol.: Mech. Dis. 2007, 2, 145, DOI: 10.1146/annurev.pathol.2.010506.092049.

[6] T. Kamura, D. M. Koepp, M. N. Conrad, D. Skowyra, R. J. Moreland, O. Iliopoulos, W. S. Lane, W. G. Kaelin, S. J. Elledge, R. C. Conaway, J. W. Harper, J. W. Conaway, Science 1999, 284, 657, DOI: 10.1126/science.284.5414.657.

[7] W.-C. Hon, M. I. Wilson, K. Harlos, T. D. W. Claridge, C. J. Schofield, C. W. Pugh, P. H. Maxwell, P. J. Ratcliffe, D. I. Stuart, E. Y. Jones, Nature 2002 417, 975, DOI: 10.1038/nature00767.

[8] a) K. Kondo, W. Y. Kim, M. Lechpammer, W. G. Kaelin, Jr., PLOS Biology 2003, 1, e83, DOI: 10.1371/journal.pbio.0000083; b) D. Schönenberger, S. Harlander, M. Rajski, R. A. Jacobs, A.-K. Lundby, M. Adlesic, T. Hejhal, P. J. Wild, C. Lundby, I. J. Frew, Cancer Res. 2016, 76, 2025, DOI 10.1158/0008-5472.can-15-1859.

[9] D. L. Buckley, J. L. Gustafson, I. Van Molle, A. G. Roth, H. S. Tae, P. C. Gareiss, W. L. Jorgensen, A. Ciulli, C. M. Crews, Angew. Chem. Int. Ed. 2012, 51, 11463, DOI: 10.1002/anie.201206231.

[10] D. B. Mendel, A. D. Laird, X. Xin, S. G. Louie, J. G. Christensen, G. Li, R. E. Schreck, T. J. Abrams, T. J. Ngai, L. B. Lee, L. J. Murray, J. Carver, E. Chan, K. G. Moss, J. Ö. Haznedar, J. Sukbuntherng, R. A. Blake, L. Sun, C. Tang, T. Miller, S. Shirazian, G. McMahon, J. M. Cherrington, Clin. Cancer Res. 2003, 9, 327.

[11] J. An, Y. Sun, M. Fisher, M. B. Rettig, Mol. Cancer Therap. 2004, 3, 727.

[12] G. V. Kondagunta, B. Drucker, L. Schwartz, J. Bacik, S. Marion, P. Russo, M. Mazumdar, R. J. Motzer, J. Clin. Oncol. 2004, 22, 3720, DOI: 10.1200/ JCO.2004.10.155.

[13] P. G. Corn, BMC Biochem. 2007, 8 Suppl 1, S4, DOI: 10.1186/1471-20918-S1-S4.

[14] Y.-J. J. Jennifer S. Isaacs, E. G. Mimnaugh, A. Martinez, F. Cuttitta, L. M. Neckers, J. Biol. Chem. 2002, 277, 29936.

[15] C. C. Hudson, M. Liu, G. G. Chiang, D. M. Otterness, D. C. Loomis, F Kaper, A. J. Giaccia, R. T. Abraham, Mol. Cell Biol. 2002, 22, 7004, DOI: 10.1128/mcb.22.20.7004-7014.2002.

[16] J. Callis, Arabidopsis Book 2014, 12, e0174, DOI: 10.1199/tab.0174.

[17] P. G. Corn, BMC Biochem. 2007, 8 Suppl 1, S4, DOI: 10.1186/1471-2091-8S1-S4.
[18] C. Capoulade, B. B.-d. Paillerets, I. Lefrère, M. Ronsin, J. Feunteun, T. Tursz, J. Wiels, Oncogene 1998, 16, 1603, DOI: 10.1038/sj.onc.1201702.

[19] L. T. Vassilev, B. T. Vu, B. Graves, D. Carvajal, F. Podlaski, Z. Filipovic, N. Kong, U. Kammlott, C. Lukacs, C. Klein, N. Fotouhi, E. A. Liu, Science 2004, 303, 844, DOI: $10.1126 /$ science. 1092472.

[20] a) A. C. Lai, C. M. Crews, Nat. Rev. Drug Discov. 2017, 16, 101, DOI: 10.1038/nrd.2016.211; b) I. Churcher, J. Med. Chem. 2018, 61, 444, DOI: 10.1021/acs.jmedchem.7b01272; c) M. Pettersson, C. M. Crews, Drug Discov. Today: Technol. 2019, 31, 15, DOI: https://doi.org/10.1016/j. ddtec.2019.01.002.

[21] D. P. Bondeson, A. Mares, I. E. D. Smith, E. Ko, S. Campos, A. H. Miah, K. E. Mulholland, N. Routly, D. L. Buckley, J. L. Gustafson, N. Zinn, P. Grandi, S. Shimamura, G. Bergamini, M. Faelth-Savitski, M. Bantscheff, C. Cox, D. A. Gordon, R. R. Willard, J. J. Flanagan, L. N. Casillas, B. J. Votta, W. den Besten, K. Famm, L. Kruidenier, P. S. Carter, J. D. Harling, I. Churcher, C. M. Crews, Nat. Chem. Biol. 2015, 11, 611, DOI: 10.1038/nchembio. 1858.

[22] P. M. Cromm, C. M. Crews, Cell Chem. Biol. 2017, 24, 1181, DOI: 10.1016/j. chembiol.2017.05.024.

[23] N. E. A. Chessum, S. Y. Sharp, J. J. Caldwell, A. E. Pasqua, B. Wilding, G. Colombano, I. Collins, B. Ozer, M. Richards, M. Rowlands, M. Stubbs, R. Burke, P. C. McAndrew, P. A. Clarke, P. Workman, M. D. Cheeseman, K. Jones, J. Med. Chem. 2018, 61, 918, DOI: 10.1021/acs.jmedchem.7b01406.

[24] S. Gu, D. Cui, X. Chen, X. Xiong, Y. Zhao, Bioessays 2018, 40, 1700247, DOI: $10.1002 /$ bies.201700247.

[25] R. P. Wurz, K. Dellamaggiore, H. Dou, N. Javier, M.-C. Lo, J. D. McCarter, D. Mohl, C. Sastri, J. R. Lipford, V. J. Cee, J. Med. Chem. 2018, 61, 453, DOI: 10.1021/acs.jmedchem.6b01781.

[26] L.-W. Xia, M.-Y. Ba, W. Liu, W. Cheng, C.-P. Hu, Q. Zhao, Y.-F. Yao, M.-R. Sun, Y.-T. Duan, Future Med. Chem. 2019, 11, 2919, DOI: 10.4155/fmc2019-0159.

[27] L. Liang, D. Astruc, Coord. Chem. Rev. 2011, 255, 2933, DOI: https://doi. org/10.1016/j.ccr.2011.06.028

[28] B. T. Worrell, J. A. Malik, V. V. Fokin, Science 2013, 340, 457, DOI: 10.1126/science. 1229506

[29] Y. Westermaier, X. Barril, L. Scapozza, Methods 2015, 71, 44, DOI: https:// doi.org/10.1016/j.ymeth.2014.08.001.

[30] D. P. Bondeson, B. E. Smith, G. M. Burslem, A. D. Buhimschi, J. Hines, S. Jaime-Figueroa, J. Wang, B. D. Hamman, A. Ishchenko, C. M. Crews, Cell Chem. Biol. 2018, 25, 78, DOI: 10.1016/j.chembiol.2017.09.010.

[31] a) M. J. Clague, C. Heride, S. Urbé, Trends in Cell Biol. 2015, 25, 417, DOI: 10.1016/j.tcb.2015.03.002; b) L. Liu, D. R. Damerell, L. Koukouflis, Y. Tong, B. D. Marsden, M. Schapira, Bioinformatics 2019, 35, 2882, DOI: 10.1093/bioinformatics/bty1067.

[32] R. P. Nowak, S. L. DeAngelo, D. Buckley, Z. He, K. A. Donovan, J. An, N. Safaee, M. P. Jedrychowski, C. M. Ponthier, M. Ishoey, T. Zhang, J. D. Mancias, N. S. Gray, J. E. Bradner, E. S. Fischer, Nat. Chem. Biol. 2018, 14, 706, DOI: $10.1038 / \mathrm{s} 41589-018-0055-\mathrm{y}$.

[33] Scott J. Hughes, A. Ciulli, Essays in Biochem. 2017, 61, 505, DOI: 10.1042/ ebc20170041.

[34] R. D. Roy, C. Rosenmund, M. I. Stefan, BMC Syst. Biol. 2017, 11, 74, DOI: 10.1186/s12918-017-0447-8.

[35] X. Zhang, V. M. Crowley, T. G. Wucherpfennig, M. M. Dix, B. F. Cravatt, Nat. Chem. Biol. 2019, 15, 737, DOI: 10.1038/s41589-019-0279-5.

[36] M. Schapira, M. F. Calabrese, A. N. Bullock, C. M. Crews, Nat. Rev. Drug Discov. 2019, 18, 949, DOI: 10.1038/s41573-019-0047-y.

\section{License and Terms}

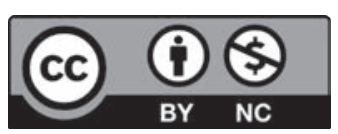

This is an Open Access article under the terms of the Creative Commons Attribution License CC BY_NC 4.0. The material may not be used for commercial purposes.

The license is subject to the CHIMIA terms and conditions: (http:// chimia.ch/component/sppagebuilder/?view=page \&id=12).

The definitive version of this article is the electronic one that can be found at doi:10.2533/chimia.2020.274 